\title{
Ion-ion correlations across and between electrified graphene layers
}

Trinidad Mendez-Morales, ${ }^{1,2,3}$ Mario Burbano, ${ }^{1,2,3}$ Matthieu Haefele, ${ }^{1}$ Benjamin Rotenberg, ${ }^{2,3}$ and Mathieu Salanne $e^{1,2,3, a)}$

1) Maison de la Simulation, CEA, CNRS, Univ. Paris-Sud, UVSQ, Université Paris-Saclay, 91191 Gif-sur-Yvette, France

2) Sorbonne Université, CNRS, Physico-chimie des électrolytes et nanosystèmes interfaciaux, PHENIX, F-75005 Paris, France

${ }^{3)}$ Réseau sur le Stockage Électrochimique de l'Énergie (RS2E), FR CNRS 3459, France

When an ionic liquid adsorbs onto a porous electrode, its ionic arrangement is deeply modified due to a screening of the Coulombic interactions by the metallic surface and by the confinement imposed upon it by the electrode's morphology. In particular, ions of the same charge can approach at close contact, leading to the formation of a superionic state. The impact of an electrified surface placed between two liquid phases is much less understood. Here we simulate a full supercapacitor made of the 1-butyl-3-methylimidazolium hexafluorophosphate and nanoporous graphene electrodes, with varying distances between the graphene sheets. The electrodes are held at constant potential by allowing the carbon charges to fluctuate. Under strong confinement conditions, we show that ions of the same charge tend to adsorb in front of each other across the graphene plane. These correlations are allowed by the formation of a highly localized image charge on the carbon atoms between the ions. They are suppressed in larger pores, when the liquid adopts a bilayer structure between the graphene sheets. These effects are qualitatively similar to the recent templating effects which have been reported during the growth of nanocrystals on a graphene substrate.

\section{INTRODUCTION}

Economic growth is directly coupled with the increase of energy consumption. In this context, society is facing a double energy challenge in the 21st century: a global transition from fossil fuels to renewable energies motivated not only by the limit on the supply but also by the environmental impacts, and the development of new devices with an enhanced ability to store energy. With this in mind, it is not surprising that supercapacitors and batteries have attracted considerable attention from research agencies and industry due to their different properties and benefits for different applications. They differ in the way they store energy: Whereas supercapacitors use a reversible adsorption of the ions on the electrode surfaces with no charge transfer, batteries rely on a reversible redox reaction that occurs in the bulk of the electrode. As a consequence, supercapacitors have a much higher charge and discharge rate and can survive many more cycles than batteries. Thus, supercapacitors provide a much greater power density, but they are still not able to store the same amount of energy as the bestperforming batteries, such as Li-ion ${ }^{1 / 3}$.

In the last years, tremendous research efforts have been devoted to increasing the energy density of supercapacitors while maintaining their high power capability and their long cycling life 4 . Taking into account that the stored energy in a supercapacitor can be calculated as $E=\frac{C V^{2}}{2}(C$ being the total capacitance of the device and $V$ the cell voltage), two approaches can be followed to reach the objective of improving their overall performance: either to develop new electrode materials 7 [10

\footnotetext{
${ }^{a)}$ Electronic mail: mathieu.salanne@sorbonne-universite.fr
}

with increased capacitance or to design novel electrolytes with wider potential windows 1113 to enhance the operating voltage (and theoretical studies also suggest a synergistic enhancement of the two quantities ${ }^{14 \mid 15}$ ).

Concerning the electrolytes, they strongly affect the performance of supercapacitors and many eyes are fixed on increasing their stability under applied voltage. For this purpose, room temperature ionic liquids (ILs) can be considered as very promising candidates, since these 'designer solvents' are very well-known for exhibiting some unique properties such as high thermal stability, high electrochemical stability, low vapor pressure and nontoxicity $16 \mid 17$.

When it comes to increasing the capacitance, it has been previously shown, both experimentally and computationally, that the interaction between the electrode material and the electrolyte plays a fundamental role in optimizing the adsorption of the ions $\frac{18}{20}$. For example, a big step forward was taken with the perception of the pore size effect and the ion desolvation under confinement made by Chmiola et al. ${ }^{21}$ when analyzing tetraethylammonium tetrafluoroborate (TEA$\left.\mathrm{BF}_{4}\right)$ in acetonitrile $(\mathrm{ACN})$ using carbide-derived carbons (CDCs). An anomalous increase of the capacitance when the ion size matches the pore size was not only observed in traditional electrolytes ${ }^{22}$, but also employing ILs 23124 . These findings broke with the traditional view that mesoporous electrodes $(>2 \mathrm{~nm}$ ) were necessary to allow the ions to enter the pores with their solvation shells intact ${ }^{25}$, and they drew the attention of the computational research community to gain deeper insight into the electrolyte environment at electrified surfaces $26 \cdot 29$ and under nanoconfinement ${ }^{30}+33$. Molecular dynamics (MD) simulations employing realistic structural models of CDC electrodes demonstrated the better efficiency of 
nanoporous electrodes over the planar ones ${ }^{34}$, because they avoid the occurrence of overscreening effects and they allow a denser packing of the ions inside the pores due to the electronic screening by the pore walls of the ions' Coulombic interactions. This latter effect is known as "superionic state" and it was first reported by Kondrat and Kornyshev $\sqrt{35 \mid 36}$. Vatamanu et al $\stackrel{37}{\text { also showed a no- }}$ ticeable improvement in the capacitive storage when designing the electrodes with carbon single-chain segments, due to their high content of atomically rough and curved surfaces. Moving to ILs confined in slit-like pores, several groups $\$ 38 \mid 39$ used simulations to predict oscillations of the capacitance as a function of the pore size. Further work insisted on the importance of pore length $\frac{40}{40}$ and on the necessity to account for the pore size distribution to interpret the experimental data 21 .

Based on these findings, many experimental groups have proposed graphene-based electrode materials to enhance supercapacitor performances 42 . In principle, it should provide the largest accessible surface among carbon materials since the ions can approach from both sides of the graphene plane. However the main difficulties consisted in i) avoiding the restacking of the layers when densifying the material and ii) providing a pathway for the ions to access the whole material. These difficulties were overcome by developing new synthesis methods. In particular, a supercapacitor made of the 1-ethyl3-methylimidazolium bis(trifluoromethane)sulfonimide (EMI-TFSI) electrolyte and holey graphene electrodes yielded a capacitance value of $45 \mathrm{~F} / \mathrm{g} 46$, while the same IL inside annealed microwave exfoliated graphene oxide (MEGO) reached $130 \mathrm{~F} / \mathrm{g}$. The performances of these devices were further expanded through chemical activation: for example the activated MEGO can store up to $200 \mathrm{~F} / \mathrm{g} 48$. The wide range of experimentally measured values is due to the variability in the structure of the materials, but it calls for further simulation studies to rationalize the results. Many works have simulated slit-pores, which neglects the fact that adjacent layers of liquid which are located on the two sides of a graphene sheet may interact with each other. With the aim of clarifying this aspect, here we carry out MD simulations of perforated nanoporous graphene in the IL 1-butyl-3methylimidazolium hexafluorophosphate $\left(\mathrm{BMIM}_{\mathrm{P}} \mathrm{PF}_{6}\right)$. In particular, we analyze the local structure of the ions inside the pores and we show that in the case where the IL is highly confined, significant correlations between two adjacent layers of liquid arise. This feature is reminiscent of the "graphene transparency" previously reported for the wettability of water ${ }^{49}$ and the growth of nanocrystals 50 on supported graphene, and it may have important consequences for charge storage in graphene-based supercapacitor electrodes.

\section{SIMULATION DETAILS}

MD simulations of pure BMIM-PF 6 confined between graphene-based electrodes were carried out within an NVE ensemble at an average temperature of $400 \mathrm{~K}$, which was chosen due to the high viscosity of this IL at room temperature $(109.2 \mathrm{mPa} \cdot \mathrm{s}$ at $313.2 \mathrm{~K}$ vs $7.1 \mathrm{mPa} \cdot \mathrm{s}$ at $413.2 \mathrm{~K}$, both at $1 \mathrm{~atm})^{51}$. Indeed, it has been previously shown that ion packing and hence, the capacitance, shows little changes with increasing the temperature from $298 \mathrm{~K}$ to $400 \mathrm{~K}^{52 / 53}$. Thus, the qualitative conclusions drawn in this paper can be extrapolated to systems at lower temperatures. The IL, composed of 4194 ions pairs, is represented by means of a coarse-grained model developed by Roy and Maroncelli $\frac{54}{\text {, in which the cations }}$ and anions are respectively characterized by 3 and 1 interaction sites. The potential parameters used for the carbon atoms of the electrodes were $\sigma_{C}=3.37 \AA$ and $\varepsilon_{C}=0.23 \mathrm{~kJ} / \mathrm{mol}$ following Cole and Klein $\frac{55}{\text {. The LJ }}$ parameters for dissimilar atoms were computed using the Lorentz-Berthelot mixing rules.

Each of the electrodes is composed of 21245 carbon atoms distributed into six fixed graphene layers. The five inner layers are randomly perforated with round $10 \AA$ radius holes ( 2 per layer) in order to allow the diffusion of the ions in the $\mathrm{z}$ direction (perpendicular to the surface) and the entrance of the liquid into the porous electrodes with fast charging and discharging rates 5 . With the aim of analyzing the effect of the slit-pore width on the capacitive properties of the system, two distances between consecutive graphene layers ( $7 \AA$ and $10 \AA$ ) were considered. The electrolyte is confined between the two electrodes as shown in Fig. 11. Periodic boundary conditions are applied along two directions, $x$ and $y$, and electrostatic interactions are calculated employing a two-dimensional Ewald summation 57 .

In order to obtain a realistic description of the properties of these devices, the simulations were carried out by holding the electrodes at constant potential $\stackrel{58}{ }$. The model, developed by Reed et al. ${ }^{[57}$ based on the previous work of Siepmann and Sprik ${ }^{59}$, uses fluctuating charges on each carbon atom that are calculated at each time step by requiring that the potential on every atom is constant and equal to the specified electrode potential. To achieve this polarization of the electrodes, the charges on the electrode atoms are represented by Gaussian distributions centered on the carbons, and they are calculated by using a conjugate gradient method to minimize the expression

$$
U=\sum_{i} q_{i}(t)\left[\frac{V_{i}\left(\left\{q_{j}(t)\right\}\right)}{2}+\frac{q_{i}(t)}{\sqrt{2 \pi} \kappa}-V^{0}\right]
$$

where $q_{i}$ is the charge on an electrode atom $i$, $V_{i}\left(\left\{q_{j}(t)\right\}\right)$ is the potential felt by an electrode atom at position $i$ due to the other electrode atoms and all the ions in the electrolyte, $\kappa$ is the width of the Gaussian 


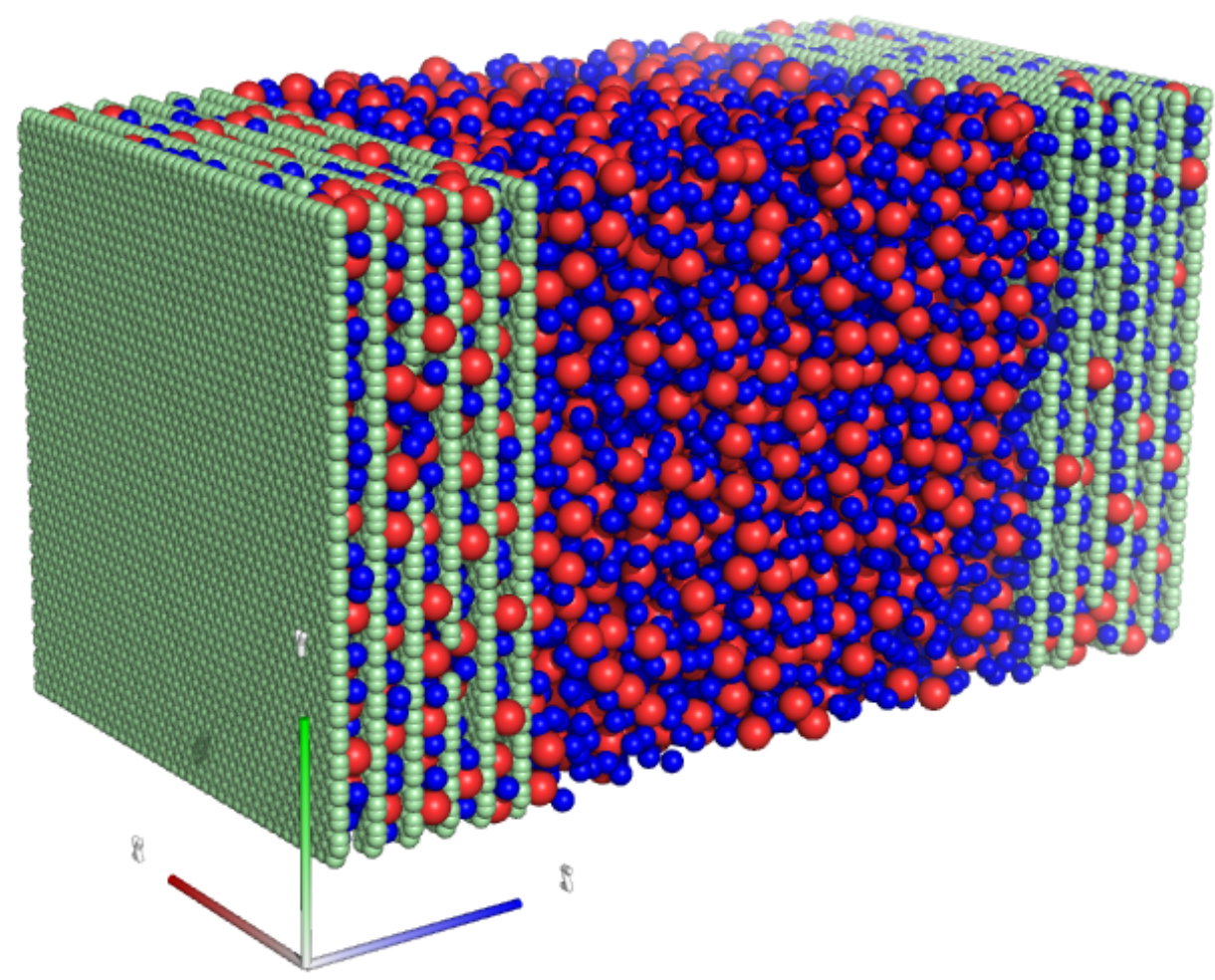

FIG. 1. Snapshot of the simulation box: $\mathrm{BMIM}^{+}$cations (blue) and $\mathrm{PF}_{6}^{-}$anions (red) confined between two graphene-based electrodes (green) with a pore size of $7 \AA$. A coarse-grained model is used for the electrolyte. (C)Trinidad Mendez-Morales, available at http://dx.doi.org/10.6084/m9.figshare.5746737.v1 under a CC-BY4.0 license.

distributions $\left(\kappa=0.5055 \AA\right.$ in our case), and $V^{0}$ is the potential applied to the electrode.

The systems were first equilibrated at constant charge during $500 \mathrm{ps}$, where the charge of the electrodes was fixed at 0 . Then we performed a second equilibration of $500 \mathrm{ps}$, in which the charges of the carbon atoms were set to $+0.01 e$ and $-0.01 e$ in the left and the right electrodes, respectively. We then carried out production runs (with a time step $d t=2 \mathrm{fs}$ ) for around $1.3 \mathrm{~ns}$ using the constant applied potential method. Since this applied potential can not be chosen by calculating the Poisson potential across the cell for the constant charge runs $\frac{60}{60}$, we performed several short simulations with different starting potentials and we chose the one for which the total charge on the electrodes showed to be nearly constant: This yielded values of $\Delta \Psi=1.8 \mathrm{~V}$ and $2.7 \mathrm{~V}$ for the applied potential for the $7 \AA$ and $10 \AA$ pore sizes, respectively. All the comparisons are thus performed for almost similar electrode charges, which is more meaningful since the ionic charge excess is then identical.

\section{RESULTS AND DISCUSSION}

\section{A. Capacitance}

The gravimetric capacitance of the simulated supercapacitor is given by

$$
C_{m}=\frac{1}{m} \frac{\left\langle Q_{+}\right\rangle}{\Delta \Psi}
$$

where $\left\langle Q_{+}\right\rangle$is the average charge of the positive electrode along the full simulation and $m$ is its corresponding mass. However, in order to compare our results to experimental data, it would be necessary to determine single electrode capacitances, which is difficult because we cannot calculate the Poisson potential across the cell for such complex pore geometries ${ }^{60}$. Nevertheless, our previous estimations in the case of CDC electrodes showed that for such a relatively simple IL (in particular with a relative symmetry for the ionic sizes), the two electrodes had almost similar capacitances. We will therefore assume that $C_{m}^{+} \approx C_{m}^{-} \approx 2 C_{m}$ for comparison purposes. We then obtain individual capacitances of 96 and $64 \mathrm{~F} / \mathrm{g}$ for the pore sizes of 7 and $10 \AA$, respectively. This falls well within the range of measured values in non-activated graphene-based supercapacitors (i.e. between 45 and 130 $\mathrm{F} / \mathrm{g} 46 \mid 47$ as discussed above). 

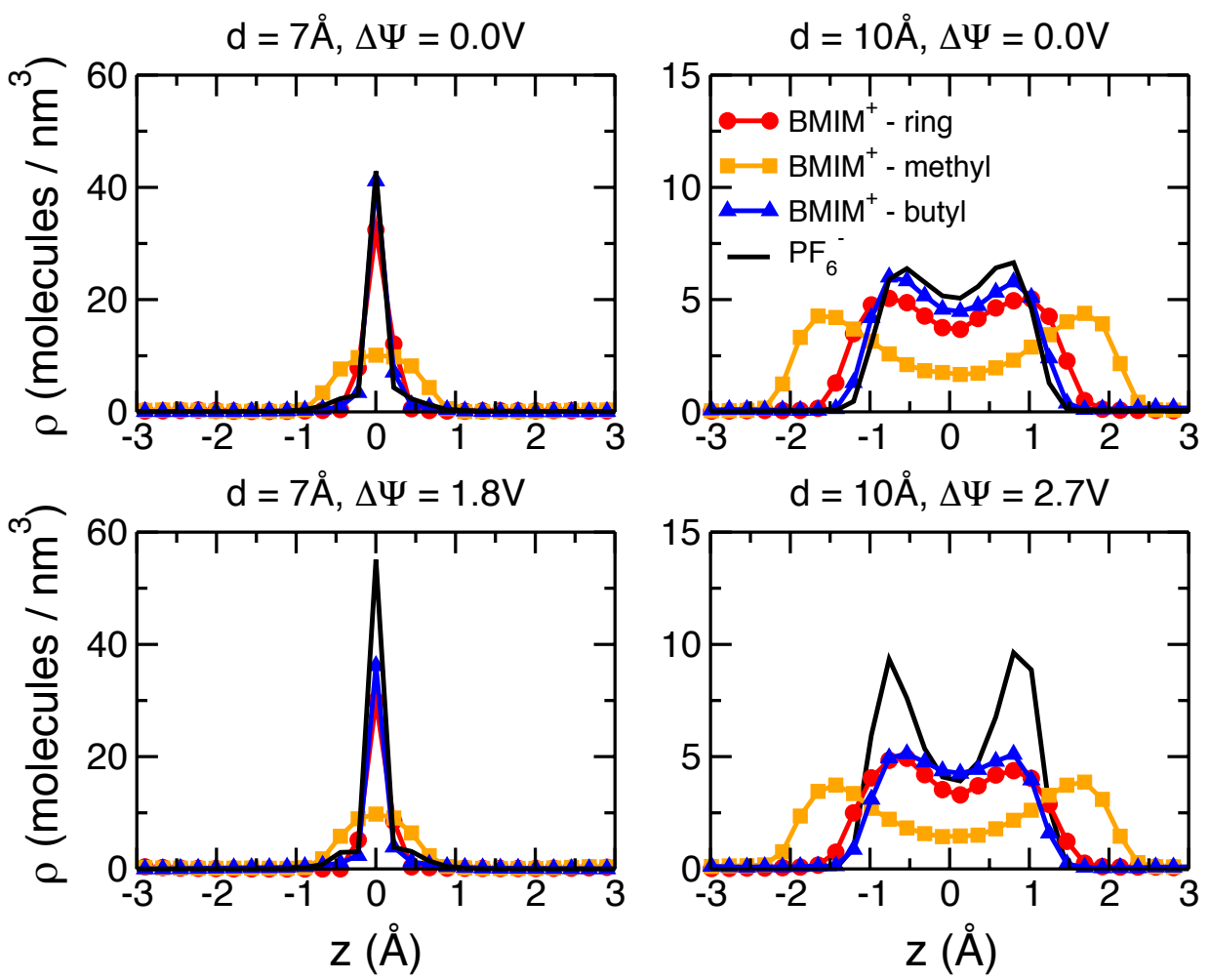

FIG. 2. Number density profiles inside the central pore of a neutral (top) and positive (bottom) electrodes, in the direction perpendicular to the electrodes (the $z$ coordinate is centered in the middle of the pore). $d$ is the pore size (7 $\AA$ in the left column and $10 \AA$ in the right one) and $\Delta \Psi$ the applied potential. (C)Trinidad Mendez-Morales and Mathieu Salanne, available at http://dx.doi.org/10.6084/m9.figshare.5746737.v1 under a CC-BY4.0 license.

\section{B. Layering inside the pores}

In previous studies performed on single slit-pore electrodes, an increase of the capacitance was observed when decreasing the pore size down to the ionic dimensions $32[38|39| 61$. This result was attributed to the transition from a monolayer to a bilayer structure for the adsorbed IL. To check whether the differences in the capacitance we observe between the two pore sizes arise from similar effects, we analyzed the structure of the IL inside each of the pores. Number densities $\left(\AA^{-3}\right)$ of the ions along the $\mathrm{z}$-direction normal to the electrodes were calculated in both cases and the profiles obtained in the neutral and positive electrodes are shown in Fig. 2. When the width of the pore is $7 \AA$, we observe that the strong confinement of the walls forces the cations and the anions to occupy a single narrow layer in the center of the pores of both electrodes (Fig. 2a), with only the smaller methyl groups of cations approaching closer to the surface. Since the neutral electrodes (i.e. for an applied potential of $0 \mathrm{~V}$ ) are already wet by the IL, applying a potential difference leads to a greater intercalation of counter-ions inside the electrodes and the expulsion of some co-ions from the pores, whereas the total number of ions per unit volume of the nanopores remains nearly the same. In this case, the IL is organized in a tight configuration that impedes an increase in ion density upon increasing the applied potential, due to which the surface charge accumulation takes place by means of a swapping mechanism. In this case, even though the coions are in direct contact with the charged surface due to the monolayer confinement, we did not observe a regime of complete intra-pore co-ion depletion.

On the contrary, in the widest pore $(10 \AA)$, the distribution of the ions is markedly different (Fig. 2b). In this case, when the system is not charged the ions accumulate in two separate layers near the pore walls, since the greater available space in the pores allows larger fluctuations in the structural distribution of the ions. It must be noted that the total number of ions adsorbed into the electrodes (given by the integral of the density) increases only slightly with the increasing pore size; which indicates that the smaller the pores, the more tightly packed the ions 35 . The bilayer structure therefore does not consist of two monolayers. Once we apply a potential difference the ion population inside the positive electrode changes in the same way as noted above (ion exchange) but now, there is also a rearrangement of anions that tend to approach closer to the surface. The minimum between the two anionic density peaks is thus more pronounced than in the neutral electrode.

In the negative electrode, the charging mechanism for 


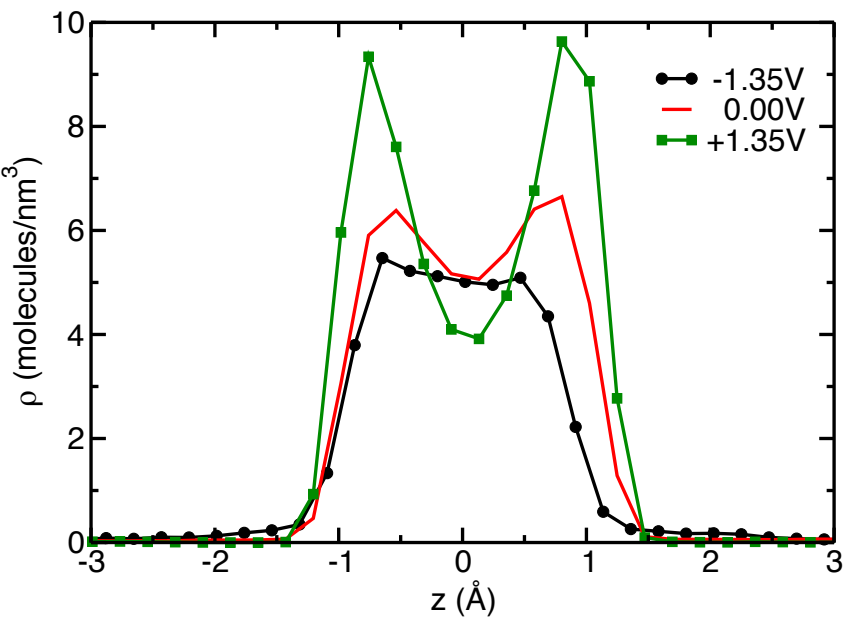

FIG. 3. Number density profiles for the anions adsorbed in the central pore of the $10 \AA$ pore size electrode, for applied potentials of $0 \mathrm{~V}$ (red) and $2.7 \mathrm{~V}$ (black: negative electrode, green: positive electrode). (c)Trinidad Mendez-Morales and Mathieu Salanne, available at http://dx.doi.org/10.6084/ m9.figshare.5746737.v1 under a CC-BY4.0 license.

\begin{tabular}{lcc}
\hline & Anion-Cation Cation-Anion \\
\hline & posit. elec. & negat. elec. \\
\hline CDC $(1.0 \mathrm{~V})$ & 2.6 & 2.5 \\
$d=7 \AA(0.0 \mathrm{~V})$ & 2.9 & 2.7 \\
$d=7 \AA(1.8 \mathrm{~V})$ & 2.3 & 2.2 \\
$d=10 \AA(2.7 \mathrm{~V})$ & 2.3 & 2.3 \\
\hline
\end{tabular}

TABLE I. Coordination numbers of cations surrounding anions in the positive electrode (left), and anions surrounding cations in the negative electrode (right). The results in CDCs were extracted from reference 63. For comparison, the bulk values for $\mathrm{A}-\mathrm{C}$ and $\mathrm{C}-\mathrm{A}$ are equal to 4.8 .

the $7 \AA$ pores is almost symmetrical with respect to the positive one. This is not the case for the $10 \AA$ pores where we observe that almost all the anions concentrate in the middle of the pores, thus losing their bilayer structure, as shown on Fig. 3 This is reminiscent of the structure which was reported for the same IL adsorbed inside CDC electrodes 58 , in which the co-ions tend to occupy the center of the pores, but also to the case of an IL adsorbed in the interlayer of negatively charged clays 52 . Again, we observe that the structure of this monolayer of anions differs markedly from the one observed in the $7 \AA$ pores since the corresponding peak in the density is much wider and less high.

\section{Decoordination inside the pores}

As it was previously reported 22 , a partial desolvation of the ions occurs under confinement (or rather a decoordination in solvent-free ILs). The associated loss of stability is compensated by the charge of the electrodes and it has been shown to be directly related with the maximum capacitance for pores smaller than the solvated ion size ${ }^{2164}$. In Table I we can observe that the coordination numbers of cations around anions (left) and vice versa (right) decreased from 4.8 in the bulk to less than 3 inside the nanopores. The coordination numbers in each electrode were calculated by averaging the number of co-ions that were closer to a central counter-ion than $7.5 \AA$, which is the first minimum of the anion-cation radial distribution function ( $\mathrm{RDF})$ in the bulk. When compared with $\mathrm{CDC} \sqrt{63}$, the ions confined in perforated graphene-based electrodes are, on average, less coordinated. It must be noted that although the distributions of the coordination numbers are centered around 2 in both cases, in the CDC case occurrences of up to 7 are observed, which is due to the presence of different adsorption sites ${ }^{63}$. As expected, a greater desolvation of the counter-ions is obtained inside charged electrodes. However, we did not register an influence of the pore size on the coordination numbers, because even though the ion density is greater in the $7 \AA$-system, the $2 \mathrm{D}$-solvation of the ions is characterized by larger lateral distances between the co-ions remaining inside the pores and the counter-ions introduced into them 51 .

\section{Partial breaking of the Coulombic ordering}

Our results suggest the stabilization of the partially desolvated counter-ions inside the nanopores by means of a charge compensation from the electrodes, which could be explained by the picture reported by Kondrat and Kornyshev $\sqrt{35136}$ of an exponential screening of the electrostatic interactions in a slit-like metallic pore due to the image charges. This superionic state breaks the Coulombic ordering characteristic of ILs and allows more ions of the same sign to occupy the pore, which leads to a high concentration (mainly limited by steric repulsions) of partially desolvated counter-ions that can be in contact with the charged walls. The screening of the electrostatic interactions is expected to sharply increase in narrower pores, thus yielding a significant improvement of the capacitive properties. This theoretical finding has also recently been confirmed experimentally by performing X-ray scattering experiments to resolve the structure of the EMI-TFSI ionic liquid confined inside electrified nanoporous carbons $\frac{65}{}$. To analyze the presence of such a dense ionic state in our simulation, we computed the ratio of the number of anion-anion (cation-cation) pairs in the positive (negative) electrode to the number of anions (cations) in that electrode. We considered the formation of a pair of ions with similar charges if they are located closer than $5.8 \AA$, which is the distance at which the anion-anion (or cation-cation) and anion-cation RDFs (both in the bulk) intersect each other (for cations the interaction center corresponding to the imidazolium ring was used to perform this analysis). A set of such pairs is 


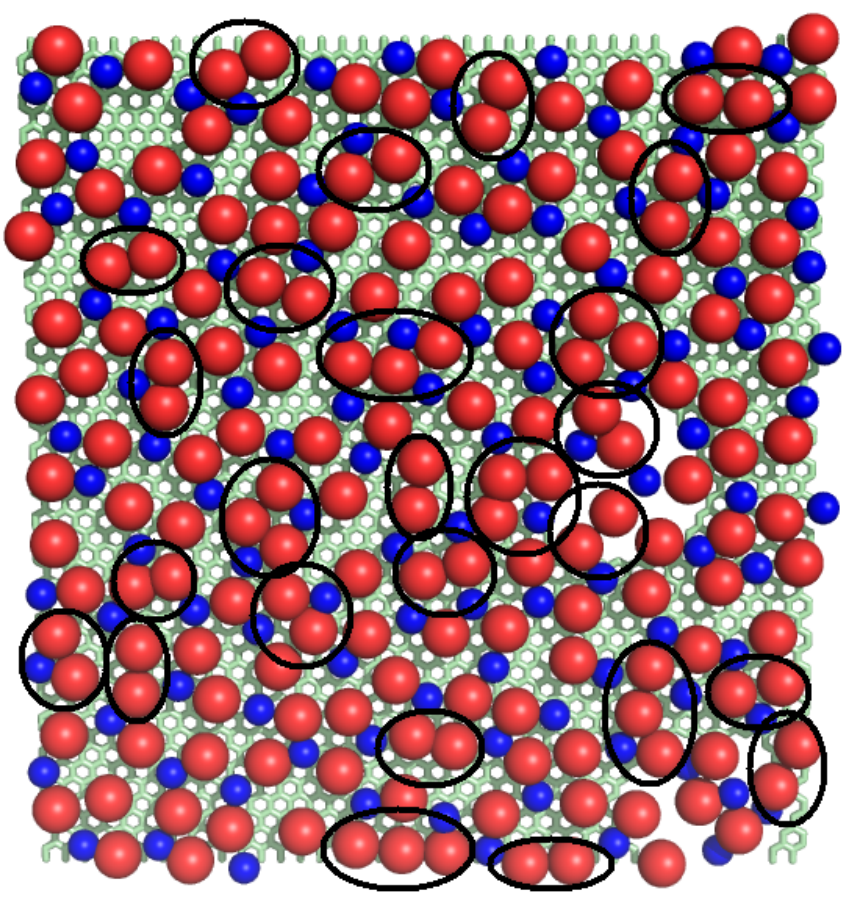

FIG. 4. Illustration of the breaking of the Coulombic ordering. Snapshot of an adsorbed layer in the positively charged $7 \AA$ pore (blue: imidazolium ring of the cations, red: anions). Pairs (and triplets) of co-ions are surrounded by black ovals. (C)Trinidad Mendez-Morales, available at http://dx.doi.org/10.6084/m9.figshare.5746737.v1 under a CC-BY4.0 license.

highlighted on a snapshot of the central adsorbed layer in the positively charged $7 \AA$ pore on Figure 4 . We can observe that the fractions increase with the confinement, switching from 0.049 to 0.109 for anions in the positive electrode and from 0.099 to 0.141 for cations in the negative one. Albeit on a different system, these numbers are in good agreement with the ones reported in the experimental study of Futamura et al ${ }^{65}$, thus providing further evidence for the existence of the superionic state for ionic liquids adsorbed inside ultranarrow electrified nanopores.

\section{E. Ion-ion correlations from pore to pore}

The results described above concerning the formation of mono/bilayer(s), decoordination and the formation of pairs of anions or cations are in agreement with the previous literature on single slit pore simulations. However, our original simulation setup allows the study ionic interactions from one pore to another. In a recent work, a templating effect of graphene was recently reported by Chae et al. 50 . They showed that the atomic arrangements of $\mathrm{ZnO}$ nanocrystals nucleated on a graphene plane exhibit a close match with those of a substrates bound to the other side of the graphene layer. In our case a simple visualization of the atomic positions in two adjacent pores (Figure 5a) suggests that similar mechanisms may be at play. In order to investigate the existence of such correlations between ions adsorbed in adjacent pores, we compute the two-dimensional radial distribution function (RDF) between an ion within a given interlayer and the ions in a neighbouring one. As illustrated on Figure 50 and c, we project an anion $I$ with coordinates $\left(x_{I}, y_{I}, z_{I}\right)$ from the layer $i$ to the layer $j$. The coordinates of this projection are then $\left(x_{I}, y_{I}, z_{I}+d\right)$ where $d$ is the pore size. We then define an in-plane distance between this projected anions and any anion $J$ present inside layer $j$ as

$$
r_{I J}^{\prime}=\sqrt{\left(x_{I}-x_{J}\right)^{2}+\left(y_{I}-y_{J}\right)^{2}}
$$

We then perform an ensemble average to determine the in-plane anion-anion RDF between layers $i$ and $j\left(g_{i j}\right)$ :

$$
g_{i j}\left(r^{\prime}\right)=\frac{S}{N_{I} N_{J} 2 \pi r^{\prime} d r^{\prime}}\left\langle\sum_{I \in i} \sum_{J \in j} \delta\left(r^{\prime}-r_{I J}^{\prime}\right)\right\rangle
$$

where $N_{I}$ and $N_{J}$ are the number of anions inside layers $i$ and $j$, respectively, $S$ is the lateral surface of the simulation cell (along $x$ and $y$ coordinates) and $d r^{\prime}$ is the precision chosen for binning the Kronecker function $\delta$. Similar RDFs can be defined for cation-cation and anion-cation pairs.

Examples of such functions are provided for various simulation setups in Figure 6 for the correlations between the fourth and fifth layers of liquid (similar results were obtained for all the adjacent layers). Firstly, very large differences are observed between the electrified pores with a size of $7 \AA$ (top) and $10 \AA$ (middle). Indeed, for the former we observe a strong peak at $r=0 \AA$ for the anion-anion, which shows that anions have a preference for adsorbing in front of other anions. On the contrary, the cation population is depleted in such sites as can be seen from the low value taken by the anion-cation RDF. The cation-cation RDF is qualitatively similar, albeit its shape is smoother due to the greater flexibility that is allowed to the $\mathrm{BMIM}^{+}$in the coarse-grained model. The structure of each fluid layer is therefore clearly correlated with that in the neighbouring ones across graphene sheets, with fluctuations due to the temperature and the presence of holes inside the graphene plane.

In the $10 \AA$ pores, these correlations are almost completely lost. A small preference for like-charge ions is still observed since the anion-anion and cation-cation RDFs slightly rise above 1 for short distances, but this effect is almost not noticeable. It is therefore necessary to have a well-defined single layer of ions to observe the effect.

In order to check whether these correlations arise from the image charges induced by the ions themselves on the surface of the carbon, we performed an additional simulation for the $7 \AA$ pores where the average charge of the plane was equally shared between all the carbon atoms. This situation is often called "constant charge" simulation, although it does not correspond to a case relevant to 

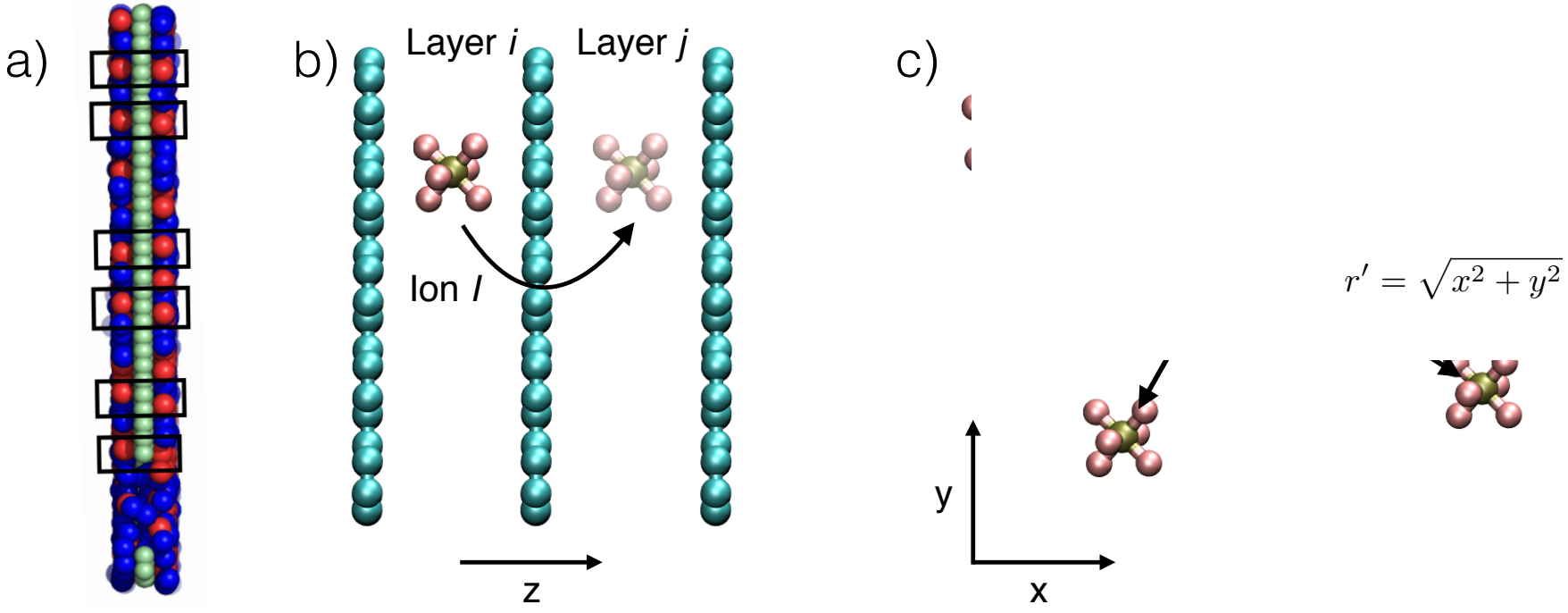

FIG. 5. a) Snapshot of a a portion of the system representing two adjacent pores separated by a graphene sheet for the simulation performed with $d=7 \AA$ and $\Delta \Psi=1.8 \mathrm{~V}$ (red: anions, blue: imidazolium rings of the cations, green: carbon atoms). b) Projection of the ion $I$ from layer $i$ to the layer $j$. c) The in-plane distances $r^{\prime}$ between the projected ion and all the ions in layer $j$ are then computed. Note that panels b) and c) show all the atoms in the $\mathrm{PF}_{6}^{-}$anion, but a coarse-grained model was used in the simulation. (c)Trinidad Mendez-Morales and Mathieu Salanne, available at http: //dx.doi.org/10.6084/m9.figshare.5746737.v1 under a CC-BY4.0 license.

electrochemical simulations. As shown in the corresponding RDFs (bottom panel of Figure 6), the correlations are then lost. On the contrary, Coulombic ordering is recovered and cations have a slight tendency to lie in front of anions from adjacent layers. This shows that the charge localization on the graphene surface plays a key role and that constant charge simulations should be avoided when simulating complex porous carbons in which the surface is accessible from the two sides.

\section{CONCLUSIONS}

In summary, BMIM- $\mathrm{PF}_{6}$ structure in nanoporous graphene-based electrodes was analyzed by means of MD simulations using the constant applied potential method to simulate the electrode materials. Both anions and cations were significantly desolvated inside the nanopores regardless of their width, but the way they are accommodated varied from a single narrow layer to a bilayer configuration when increasing the pore size from $7 \AA$ to $10 \AA$. Additionally, the ions were found to be more densely packed in the smaller pores, forming a single monolayer while they arrange in two layers when they have access to a larger volume. The charging of the supercapacitor is then allowed by an efficient screening of the Coulombic interactions between the ions by the image charges. This superionic state previously predicted by Kondrat and Kornyshev was confirmed by a more marked formation of counter-ion/counter-ion pairs under a strong confinement.

The main specificity of the presence of a single graphene sheet between adjacent layers of liquids is that it allows the presence of structural correlations between ions of the same sign belonging to different pores; that is, the distribution of the ions in one layer is strongly influenced by the conformation that the ions adopt inside the other layers due to the image forces. It is worth noting that in a former DFT study, it was shown that two $\mathrm{BF}_{4}^{-}$ions adsorbing on the same site on both sides of a graphene layer were more stable than those on different sites 66 . Here the correlation effects seem to be enhanced by a strong confinement since they are much more remarkable in the thinner pores, and almost absent when a bilayer of liquid is allowed to form inside the electrode. We proved that here again, the strong localization of image charges on the graphene surface plays a key role: When assigning similar constant charges to the carbon atoms (with the same average as in the constant potential simulation), the correlations are reversed and the usual Coulombic ordering is recovered. These results open further questions for the development of efficient graphene-based supercapacitors. For example, it will be of great interest to determine whether these correlations remain present in realistic models, where restacking of the graphene layers is likely to happen. Much of the applications involve organic electrolytes $\sqrt{67}$ or solvate ionic liquids ${ }^{68}$, so that it will also be of importance to determine if the presence of a solvent mitigates the importance of the ion-ion correlations across the graphene layers. 

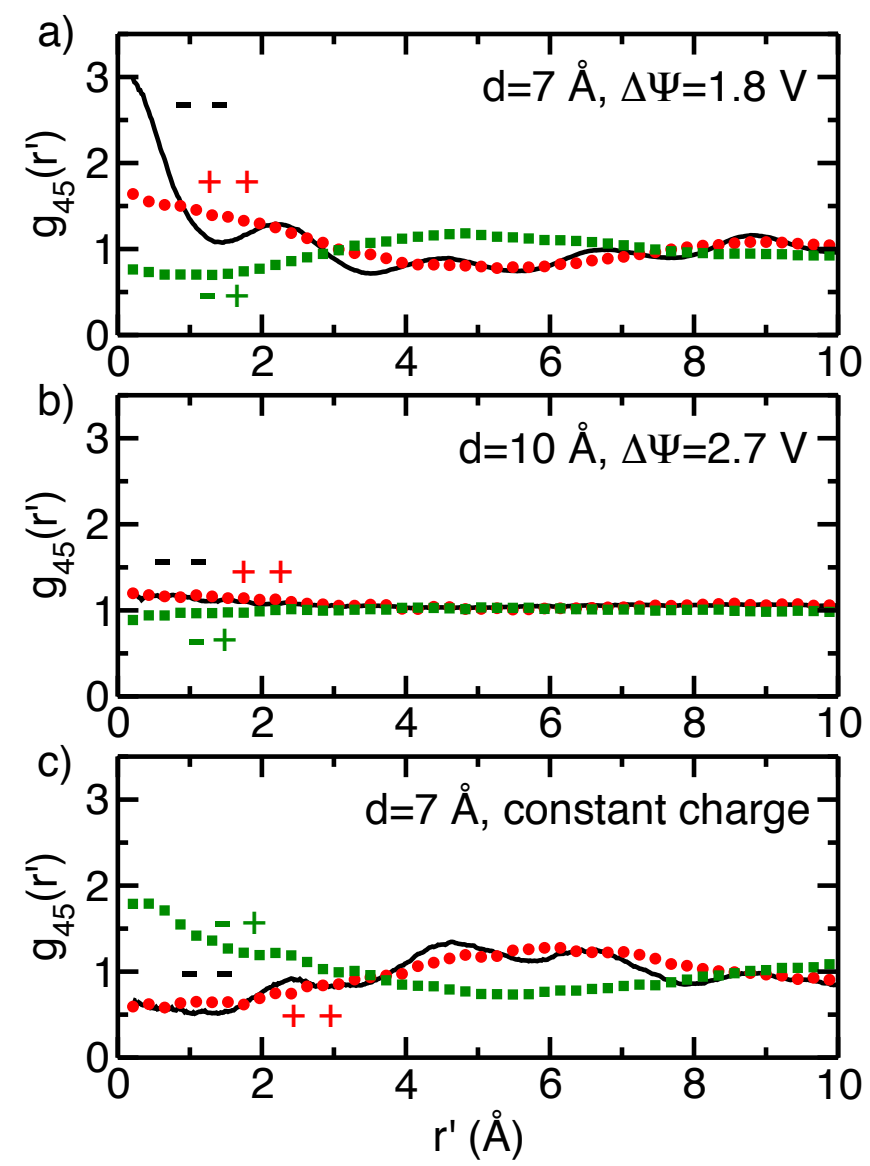

FIG. 6. In-plane radial distribution functions between an ion within the fourth layer of adsorbed liquid and the ions in the fifth one (black: anion-anion, red: cation-cation and green: anion-cation). Panels a) and b): A constant potential is applied to the electrode, allowing the surface charge to fluctuate in response to the electrolyte (a: $d=7 \AA \quad \Delta \Psi=1.8 \mathrm{~V}, \mathrm{~b}: d=$ $10 \AA \Delta \Psi=2.7 \mathrm{~V})$. Panel c): The partial charges on the carbon atoms are held constant and equal to the average charge for the $1.8 \mathrm{~V}$ applied potential simulation $(d=7 \AA)$. (C)Trinidad Mendez-Morales and Mathieu Salanne, available at http:// dx.doi.org/10.6084/m9.figshare.5746737.v1 under a CCBY4.0 license.

\section{ACKNOWLEDGMENTS}

This work was supported by the French National Research Agency (Labex STORE-EX, Grant No. ANR10-LABX-0076 and ANR SELFIE, Grant No. ANR17-ERC2-0028) and by Defi CNRS INPHYNITI 20152016 (SIMELEC). We acknowledge support from EoCoE, a project funded by the European Union Contract No. H2020-EINFRA-2015-1-676629, from the DSMnergie programme of CEA and from the Eurotalent programme. We are grateful for the computing resources on OCCIGEN (CINES, French National HPC) and CURIE (TGCC, French National HPC) obtained through the project x2016096728.
${ }^{1}$ X. Luo, J. Wang, M. Dooner, and J. Clarke, Appl. Energy 137, $511(2015)$.

${ }^{2}$ I. V. Barsukov, C. S. Johnson, J. E. Doninger, and V. Z. Barsukov, New Carbon Based Materials for Electrochemical Energy Storage Systems (Springer, 2003).

${ }^{3}$ B. E. Conway, Electrochemical Supercapacitors. Scientific Fundamentals and Technological Applications. (Kluwer Academic/Plenum Publishers, New York, 1999).

${ }^{4}$ H. J. Qiu, L. Y. Chen, Y. Ito, J. L. Kang, X. W. Guo, P. Liu, H. Kashani, A. Hirata, T. Fujita, and M. W. Chen, Nanoscale 8, 18551 (2016).

${ }^{5}$ Z. S. Wu, K. Parvez, X. Feng, and K. Mllen, Nat. Commun. 4, 1 (2013)

${ }^{6}$ T. Lin, I.-W. Chen, F. Liu, C.Yang, H. Bi, F. Xu, and F. Huang, Science 350, 1508 (2015).

${ }^{7}$ E. Frackowiak, Q. Abbas, and F. Béguin, J. Ener. Chem. 22, 226 (2013).

${ }^{8}$ P. Simon and Y. Gogotsi, Nat. Mater. 7, 845 (2008).

${ }^{9} \mathrm{Z}$. Li, J. Liu, and T. Thundat, Nano Energy 25, 161 (2016).

${ }^{10}$ J. Zhu, A. S. Childress, M. Karakaya, S. Dandeliya, A. Srivastava, Y. Lin, A. M. Rao, and R. Podila, Adv. Mater. 28, 7185 (2016).

${ }^{11}$ A. Brandt, P. Isken, A. Lex-Balducci, and A. Balducci, J. Power Sources 204, 213 (2012).

${ }^{12}$ P. Ray, S. Dohm, T. Husch, C. Schütter, K. A. Persson, A. Balducci, B. Kirchner, and M. Korth, J. Phys. Chem. C 120, 12325 (2016).

${ }^{13}$ C. Schütter, S.Passerini, M. Korth, and A. Balducci, Electrochim. Acta 224, 278 (2017).

${ }^{14}$ A. A. Lee, D. Vella, A. Goriely, and S. Kondrat, Phys. Rev. X 6, 021034 (2016).

${ }^{15}$ S. Kondrat and A. Kornyshev, Nanoscale Horiz. 1, 45 (2016).

${ }^{16}$ D. R. MacFarlane, N. Tachikawa, M. Forsyth, J. M. Pringle, P. C. Howlett, G. D. Elliott, J. H. Davis, M. Watanabe, P. Simon, and C. A. Angell, Energy Environ. Sci. 7, 232 (2014).

${ }^{17}$ T. Tsuda and C. L. Hussey, Electrochem. Soc. Interf. 16, 42 (2007).

${ }^{18}$ M. V. Fedorov and A. A. Kornyshev, Chem. Rev. 114, 2978 (2014).

${ }^{19}$ M. Salanne, B. Rotenberg, K. Naoi, K. Kaneko, P.-L. Taberna, C. P. Grey, B. Dunn, and P. Simon, Nat. Energy 1, 16070 (2016).

${ }^{20}$ A. C. Forse, C. Merlet, J. M. Griffin, and C. P. Grey, J. Am. Chem. Soc. 138, 5731 (2016).

${ }^{21}$ J. Chmiola, G. Yushin, Y. Gogotsi, C. Portet, P. Simon, and P.-L. Taberna, Science 313, 1760 (2006).

${ }^{22}$ J. Chmiola, C. Largeot, P.-L. Taberna, P. Simon, and Y. Gogotsi, Angew. Chem. Int. Ed. 47, 3392 (2008).

${ }^{23}$ C. Largeot, C. Portet, J. Chmiola, P. L. Taberna, Y. Gogotsi, and P. Simon, J. Am. Chem. Soc. 130, 2730 (2008).

${ }^{24}$ C. O. Ania, J. Pernak, F. Stefaniak, E. Raymundo-Piero, and F. Bguin, Carbon 44, 3126 (2006).

${ }^{25}$ M. Endo, T. Maeda, T. Takeda, Y. J. Kim, K. Koshiba, H. Hara, and M. S. Desselhaus, J. Electrochem. Soc. 148, A910 (2001).

${ }^{26}$ K. Kirchner, T. Kirchner, V. Ivanistsev, and M. V. Fedorov, Electrochim. Acta 110, 762 (2013).

${ }^{27}$ K. Breitsprecher, K. Szuttor, and C. Holm, J. Phys. Chem. C 119, 22445 (2015).

${ }^{28}$ V. Ivanistsev, M. V. Fedorov, and R. M. Lynden-Bell, J. Phys. Chem. C 118, 5841 (2014).

${ }^{29}$ T. Méndez-Morales, J. Carrete, M. Pérez-Rodríguez, O. Cabeza, L. J. Gallego, R. M. Lynden-Bell, and L. M. Varela, Phys. Chem. Chem. Phys. 16, 13271 (2014).

${ }^{30} \mathrm{P}$. Wu, J. Huang, V. Meunier, B. G. Sumpter, and R. Qiao, ACS Nano 5, 9044 (2011).

${ }^{31}$ Y. Shim and H. J. Kim, ACS Nano 4, 2345 (2010).

${ }^{32}$ L. Xing, J. Vatamanu, O. Borodin, and D. Bedrov, J. Phys. Chem. Lett. 4, 132 (2013).

${ }^{33}$ J. Vatamanu and D. Bedrov, J. Phys. Chem. Lett. 6, 3594 (2015).

${ }^{34}$ C. Merlet, B. Rotenberg, P. A. Madden, P.-L. Taberna, P. Simon, Y. Gogotsi, and M. Salanne, Nat. Mater. 11, 306 (2012). 
${ }^{35}$ S. Kondrat and A. A. Kornyshev, J. Phys.: Condens. Matter 23, 022201(1) (2011).

${ }^{36}$ S. Kondrat, N. Georgi, M. V. Fedorov, and A. A. Kornyshev, Phys. Chem. Chem. Phys. 13, 11359 (2011).

${ }^{37}$ J. Vatamanu, Z. Hu, and D. Bedrov, J. Phys. Chem. Lett. 4, 2829 (2013).

${ }^{38}$ G. Feng and P. T. Cummings, J. Phys. Chem. Lett. 2, 2859 (2011).

${ }^{39}$ D. Jiang, Z. Jin, and J. Wu, Nano Lett. 11, 5373 (2011).

${ }^{40}$ K. Breitsprecher, M. Abele, S. Kondrat, and C. Holm, J. Chem. Phys. 147, 104708 (2017).

${ }^{41}$ S. Kondrat, C. R. Prez, V. Presser, Y. Gogotsi, and A. A. Kornyshev, Energy Environ. Sci. 5, 6474 (2012).

${ }^{42}$ M. Stoller, S. Park, Y. Zhu, J. An, and R. Ruoff, Nano. Lett. 8, 3498 (2008).

${ }^{43}$ C. Liu, Z. Yu, D. Neff, A. Zhamu, and B. Z. Jang, Nano Lett. 10, 4863 (2010).

${ }^{44}$ J. J. Yoo, K. Balakrishnan, J. Huang, V. Meunier, B. G. Sumpter, A. Srivastava, M. Conway, A. L. M. Reddy, J. Yu, R. Vajtai, et al., Nano Lett. 11, 1423 (2011).

${ }^{45}$ G. Lian, C.-C. Tuan, L. Li, S. Jiao, K.-S. Moon, Q. Wang, D. Cui, and C.-P. Wong, Nano Lett. 17, 1365 (2017).

${ }^{46}$ X. Han, M. R. Funk, F. Shen, Y.-C. Chen, Y. Li, C. J. Campbell, J. Dai, X. Yang, J.-W. Kim, Y. Liao, et al., ACS Nano 8, 8255 (2014).

${ }^{47}$ W.-Y. Tsai, R. Lin, S. Murali, L. L. Zhang, J. K. McDonough, R. S. Ruoff, P.-L. Taberna, Y. Gogotsi, and P. Simon, Nano Ener. 2, 403 (2013).

${ }^{48}$ R. Zhu, S. Murali, M. D. Stoller, K. J. Ganesh, W. Cai, P. J. Ferreira, A. Pirkle, R. M. Wallace, K. A. Cychosz, M. Thommes, et al., Science 332, 1537 (2011).

${ }^{49}$ J. Rafiee, X. Mi, H. Gullapalli, H. V. Thomas, F. Yavari, Y. Shi, P. M. Ajayan, and N. A. Koratkar, Nat. Mater. 11, 217 (2012).

${ }^{50}$ S. Chae, S. Jang, W. J. Choi, Y. S. Kim, H. Chang, T. I. Lee, and J.-O. Lee, Nano Lett. 17, 1711 (2017).

${ }^{51}$ Y. Qiao, F. Yan, S. Xia, S. Yin, and P. Ma, J. Chem. Eng. Data
56, 2379 (2011).

${ }^{52}$ G. Feng, S. Li, J. S. Atchison, V. Presser, and P. T. Cummings, J. Phys. Chem. C 117, 9178 (2013).

${ }^{53}$ J. Vatamanu, L. Xing, W. Libc, and D. Bedrov, Phys. Chem. Chem. Phys. 16, 5174 (2014).

${ }^{54}$ D. Roy and M. Maroncelli, J. Phys. Chem. B 114, 12629 (2010).

${ }^{55}$ M. W. Cole and J. R. Klein, Surf. Sci. 124, 547 (1983).

${ }^{56}$ X. Han, M. R. Funk, Y. Chen, Y. Li, C. J. Campbell, J. Dai, X. Yang, J. Kim, Y. Liao, J. W. Connell, et al., ACS Nano 8, 8255 (2014).

${ }^{57}$ S. K. Reed, O. J. Lanning, and P. A. Madden, J. Chem. Phys. 126, 084704(1) (2007).

${ }^{58}$ C. Merlet, C. Péan, B. Rotenberg, P. A. Madden, P. Simon, and M. Salanne, J. Phys. Chem. Lett. 4, 264 (2012).

${ }^{59}$ J. I. Siepmann and M. Sprik, J. Chem. Phys. 102, 511 (1995).

${ }^{60}$ C. Pean, B. Daffos, C. Merlet, B. Rotenberg, P.-L. Taberna, P. Simon, and M. Salanne, J. Electrochem. Soc. 162, A5091 (2015).

${ }^{61}$ J. Vatamanu, M. Vatamanu, and D. Bedrov, ACS Nano 9, 5999 (2015).

${ }^{62}$ D. Duarte, M. Salanne, B. Rotenberg, M. A. Bizeto, and L. J. A. Siqueira, J. Phys.: Condens. Matter 26, 284107 (2014).

${ }^{63}$ C. Merlet, C. Pan, B. Rotenberg, P. A. Madden, B. Daffos, P. L. Taberna, P. Simon, and M. Salanne, Nat. Commun. 4, 1 (2013). ${ }^{64}$ P. Simon and Y. Gogotsi, Acc. Chem. Res. 46, 1094 (2013).

${ }^{65}$ R. Futamura, T. Iiyama, Y. Takasaki, Y. Gogotsi, M. J. Biggs, M. Salanne, J. Ségalini, P. Simon, and K. Kaneko, Nat. Mater. 16, 1225 (2017).

${ }^{66}$ Y. Kunisada, H. Kishi, F. Dimas, M. Y. David, H. Nakanishi, H. Kasai, T. Asari, and S. Hayashi, Jpn. J. Appl. Phys. 49, 02BB04 (2010).

${ }^{67}$ R. Burt, K. Breitsprecher, B. Daffos, P.-L. Taberna, P. Simon, G. Birkett, X. S. Zhao, C. Holm, and M. Salanne, J. Phys. Chem. Lett. 7, 4015 (2016).

${ }^{68}$ S. W. Coles, M. Mishin, S. Perkin, M. V. Fedorov, and V. B. Ivanistsev, Phys. Chem. Chem. Phys. 19, 11004 (2017). 